Paolo Formaggioni, Piero Franceschi, Massimo Malacarne, Andrea Summer

\title{
Effetto delle cellule somatiche e della stagione sulla resa in formaggio Parmigiano Reggiano e Grana Padano: una minireview
}

\author{
Effect of somatic cells and season on Parmigiano Reggiano and Grana Padano \\ cheese yields: a minireview
}

Dipartimento di Scienze Medico-Veterinarie, Università degli Studi di Parma, Via del Taglio 10, I-43126, Parma

*Corresponding author: Paolo Formaggioni

Dipartimento di Scienze Medico-Veterinarie, Università degli Studi di Parma

Via del Taglio 10, I-43126, Parma I Tel. 0521-032617 | Email: paolo.formaggioni@unipr.it
Ricevuto il 13 maggio 2020

Accettato il 23 giugno 2020

DOI: 10.36138/STLC.04.2020.02

\section{Riassunto}

La rassegna si propone di investigare tutti gli aspetti inerenti gli effetti delle cellule somatiche e della stagione sulla resa in formaggio Parmigiano Reggiano e Grana Padano, recensendo tutti i lavori che in letteratura si sono occupati di queste tematiche. In particolare, per quanto riguarda l'effetto delle cellule somatiche, verrà considerato: l'effetto sulla resa di latti con più di $400.000 \mathrm{cell} / \mathrm{mL}$; la resa in formaggio di 5 classi di latti con diverso valore di cellule somatiche; una spiegazione delle motivazioni principali per cui latti con cellule alte determinano una perdita di resa in formaggio. Per quanto riguarda le variazioni stagionali verranno presi in considerazione le variazioni della resa in formaggio grana registrate da Parisi nel 1966, dati più recenti sulle variazioni della resa in Parmigiano Reggiano (1990) e della resa in Grana Padano (1995), e una ricerca ancora più recente (2004) relativa alle variazioni stagionali della resa in Parmigiano Reggiano.
Parole chiave:
- Resa in formaggio
- Parmigiano Reggiano
- Grana Padano
- Fattori di variazione
- Cellule somatiche
Variazioni stagionali

\section{Abstract}

The review aims to investigate all aspects concerning the effects of somatic cells and the season on Parmigiano Reggiano and Grana Padano cheese yield, reviewing all the papers that have dealt with these issues in literature. In particular, as regards the effect of the somatic cells, the following issues will be considered: the effect on cheese yield of milks with more than 400,000 cells $/ \mathrm{mL}$; the cheese yield of 5 classes of milks with different somatic cell values; an explanation of the main reasons why milks with high somatic cells cause a loss of yield in cheese. As regards the seasonal variations, the variations in the grana cheese yield recorded by Parisi in 1966 will be taken into consideration, more recent data on the variations in the Parmigiano Reggiano cheese yield (1990) and in the Grana Padano cheese yield (1995), and an even more recent research (2004) relating to seasonal variations of the Parmigiano Reggiano cheese yield.
Keywords
- Cheese yield
- Parmigiano Reggiano
- Grana Padano
- Variation factors
- Somatic cells
- Seasonal variations 




Figura 1. Resa a 24 ore in formaggio Parmigiano Reggiano di latte con meno di 400.000 cell/mL e latte con più di $400.000 \mathrm{cell} / \mathrm{mL}$ (10 prove comparative; 10 caseifici diversi) (Summer et al., 2010; Costanzo et al. 2015).

Figure 1. 24-hours Parmigiano Reggiano cheese yield of milk with less than $400,000 \mathrm{cells} / \mathrm{mL}$ and milk with more than 400,000 cells/mL (10 comparative tests; 10 different dairies) (Summer et al., 2010; Costanzo et al. 2015).

\section{CELLULE SOMATICHE E RESA IN FORMAGGIO PARMIGIANO REGGIANO E GRANA PADANO}

I processi infiammatori della ghiandola mammaria si caratterizzano per un aumento del contenuto di cellule somatiche (leucociti) nel latte $[1,2]$. La risposta infiammatoria, inoltre, è caratterizzata da un passaggio nel latte di alcune componenti del sangue [3] e da una diminuzione dell'attività secretoria della ghiandola mammaria [4, 5]. Le bovine affette da infiammazioni del tessuto mammario manifestano, quindi, disordini secretori con conseguente riduzione della produzione di latte [6]. Inoltre, il latte prodotto è caratterizzato da alterazioni di composizione chimica [7] e delle proprietà fisico-chimiche [8], tali da comportare una riduzione della resa casearia $[9,10]$.

Latti con più di 400.000 cell/mL danno rese più basse: Summer et al. (2010) e Costanzo et al. (2015)

Summer et al. [11] e Costanzo et al. [12] hanno studiato l'influenza di 2 livelli di contenuto in cellule somatiche del latte (fino a 400.000 cellule/mL e sopra le 400.000 cellule/mL) sulla composizione chimica, sulla resa casearia e sulle perdite di sostanze nel siero cotto nella trasformazione del latte a formaggio Parmigiano Reggiano.
Nel corso di 2 anni, presso 10 caseifici in provincia di Parma, sono state effettuate 10 prove comparative di caseificazione a formaggio Parmigiano Reggiano. In ciascuna prova, sono state effettuate 2 lavorazioni in parallelo: una presentava un contenuto di cellule somatiche - rilevato sul latte della sera - basso (LCC: contenuto di cellule somatiche $<400.000$ cellule/mL), mentre l'altra si caratterizzava per un contenuto di cellule elevato (HCC: contenuto di cellule somatiche $>400.000$ ma inferiore a 1.000 .000 cellule $/ \mathrm{mL}$ ). Per ciascuna lavorazione è stato prelevato un campione di latte in caldaia.

Le cellule somatiche del latte intero della sera, nei due gruppi, risultavano mediamente 233.000 (gruppo LCC) e 538.000 (gruppo HCC). ॥ latte in caldaia LCC si è caratterizzato per maggiori valori di caseina (2,57 vs 2,43 g/100g; $P<0,05)$ e indice di caseina $(77,80$ vs $77,03 \%$; $\mathrm{P}<0,05)$ rispetto al latte in caldaia HCC. L'acidità titolabile ha mostrato valori significativamente maggiori nel latte in caldaia LCC rispetto a HCC (3,34 vs $\left.3,160{ }^{\circ} \mathrm{SH} / 50 \mathrm{~mL}, \mathrm{P}<0,05\right)$; diversamente, il $\mathrm{pH}(6,71$ vs 6,$77 ; \mathrm{P}<0,05)$ e le cellule somatiche ( 146.000 vs 259.000 cellule/ $\mathrm{mL} ; \mathrm{P}<0,001)$ sono risultati significativamente più bassi nel latte in caldaia LCC rispetto a HCC. La resa in formaggio del latte in caldaia LCC è risultata significativamente più elevata a 24 ore $(8,68$ vs 8,02 


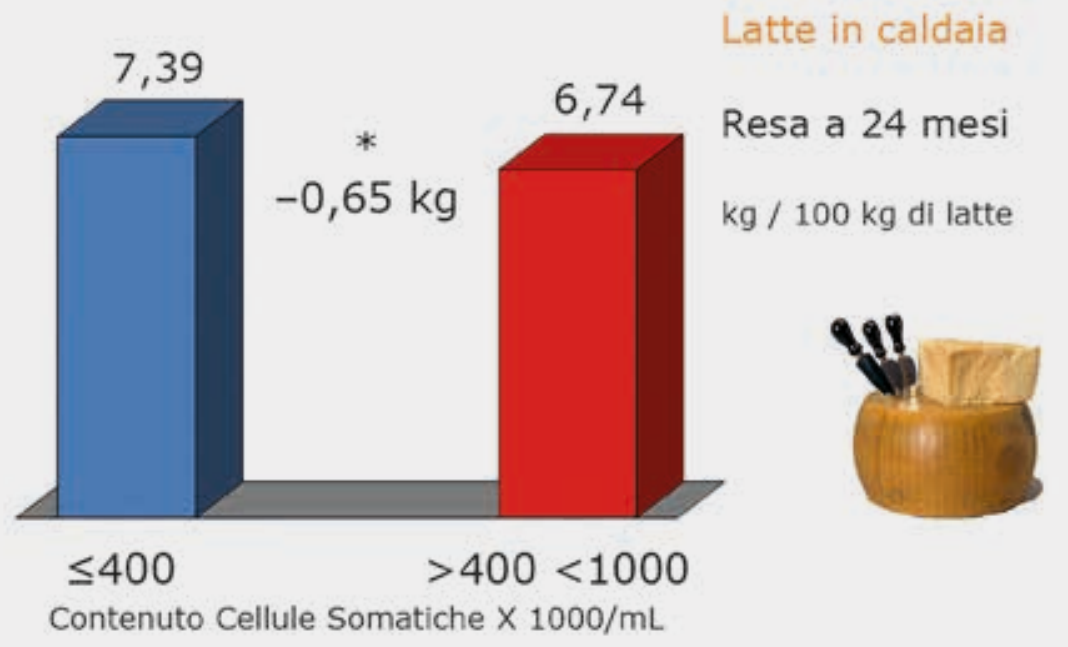

$\mathrm{kg} / 100 \mathrm{~kg}$; $\mathrm{P}<0,01)$ e a 24 mesi di stagionatura $(7,39$ vs $6,74 \mathrm{~kg} / 100 \mathrm{~kg}$; $\mathrm{P}<0,05$ ) rispetto alla resa in formaggio del latte in caldaia HCC (Figure 1 e 2). Lo stesso si è registrato per il rendimento caseario riferito al latte conferito (latte della sera intero più latte del mattino intero) del gruppo LCC, che è risultato significativamente più elevato a 24 ore ( 8,41 vs 7,77 kg/100kg; $P<0,01)$ e a 24 mesi di stagionatura $(7,16$ vs $6,55 \mathrm{~kg} / 100 \mathrm{~kg} ; \mathrm{P}<0,05)$ rispetto a quello del latte conferito HCC. Infine, anche la resa misurata a 24 mesi, riferita al residuo secco del formaggio, è risultata significativamente più elevata per il latte LCC, rispetto a HCC, sia rapportata al latte in caldaia $(5,19$ vs $4,74 \mathrm{~kg} / 100 \mathrm{~kg}$; $\mathrm{P}<0,05)$, che rapportata al latte conferito $(5,02$ vs $4,60 \mathrm{~kg} / 100 \mathrm{~kg}$; $\mathrm{P}<0,05)$. Le migliori caratteristiche del latte in caldaia con meno di 400.000 cellule $/ \mathrm{mL}$ rispetto a quello con più di 400.000 cellule $/ \mathrm{mL}$, quindi, hanno comportato una più elevata resa casearia, sia riferita al latte in caldaia $(+8,10 \%$ a 24 mesi di stagionatura), che riferita al latte conferito $(+7,61 \%$ a 24 mesi di stagionatura).

La resa in formaggio di 5 classi di latti con diverso valore di cellule somatiche: Summer et al (2015)

Lo scopo dello studio di Summer et al. [13] è stato quello di determinare l'influenza del contenuto di cellule somatiche del latte sulla resa in formaggio Parmigiano Reggiano, prodotto in caseifici commerciali in condizioni di campo. Lo studio è stato condotto seguen- do la produzione di 56 lotti di Parmigiano Reggiano in 13 caseifici commerciali che trasformavano il latte proveniente da allevamenti di bovine di razza Frisona Italiana. I lotti di formaggio prodotti sono stati divisi in 5 classi, in base al valore di cellule somatiche del latte della sera, determinato prima della scrematura naturale (classe 1 , da 0 a 200.000; 2, 201.000-300.000; 3, 301.000-400.000; 4, 401.000-500.000; 5, oltre 501.000 cellule/mL). I valori di grasso, proteina, caseina e resa a 24 ore (si intende riferita al latte in caldaia) risultavano correlati negativamente con il punteggio delle cellule somatiche (Somatic Cell Score: SCS) del latte della sera. Al contrario, è stata osservata una correlazione positiva tra cloruri e SCS. Grasso, frazioni proteiche (proteina, caseina e sieroproteine), fosforo e acidità titolabile del latte in caldaia risultavano positivamente correlati con la resa a 24 ore, mentre cloruri, pH e SCS mostravano una correlazione negativa. Un calo significativo della resa a 24 ore è stato osservato nelle classi 3, 4 e 5, ovvero quando il valore di cellule somatiche del latte della sera superava le 300.000 cellule/mL, quindi prima che il latte raggiungesse il valore di 400.000 cellule $/ \mathrm{mL}$ (limite legale europeo) (Figura 3). Infine, è stato osservato un minor recupero del grasso del latte nel formaggio con l'aumento del contenuto di cellule somatiche del latte della sera.

Le differenze osservate nella resa in formaggio possono risultare rilevanti per il profitto di un caseificio. Supponendo condizioni stan- 
dard di produzione del formaggio Parmigiano Reggiano (1100 kg di latte in caldaia trasformato, perdita del $15 \%$ del peso del formaggio durante una stagionatura di 24 mesi) e un prezzo di 9,76 euro/kg per il formaggio di 24 mesi, secondo i valori di resa osservati in questo studio, è possibile stimare una riduzione dell'utile di 46 euro per ogni lavorazione effettuata con latte con contenuto di cellule somatiche nell'intervallo $300.000-400.000$ cellule/mL rispetto ad una lavorazione effettuata con latte con contenuto di cellule somatiche inferiore a 300.000 cellule/ml.

Perché latti con cellule alte determinano una perdita di resa in formaggio

La perdita di resa, nei latti con cellule somatiche elevate, si manifesta a diversi livelli e per numerose motivazioni. Queste le principali: 1) Nel latte con elevato contenuto di cellule somatiche, la caseina risulta presente in minore quantità (minore sintesi) e in peggiori condizioni, in quanto parzialmente idrolizzata dalla plasmina e dagli enzimi rilasciati dai globuli bianchi. Non dimentichiamo che i latti ad elevati contenuti di cellule somatiche sono anche associati con un significativo aumento dell'attività della plasmina. Di conseguenza parte delle caseine si perdono nel siero come proteoso-peptoni o altri peptidi solubili. 2) II peggioramento delle proprietà di coagulazione dà luogo ad un reticolo con caratteristiche peggiori. Nella conversione di latte a formag- gio, le micelle di caseina si aggregano per formare un reticolo che intrappola la fase siero. Ogni variazione nella composizione delle micelle di caseina può perciò avere una influenza sull'efficienza di incorporazione dei componenti in questo reticolo. La diminuzione della resistenza della cagliata al momento del taglio esercita un effetto sulla resa in formaggio influenzando le perdite di grasso e caseina nel siero. 3) Lavorando latte con un più elevato contenuto di cellule somatiche i granuli caseosi risultano di dimensioni molto eterogenee. Quelli più piccoli manifestano una eccessiva "sensibilità" al "fuoco", non si asciugano in misura adeguata e perdono la loro capacità di coesione, restando nel siero e concorrono a determinare una minore efficienza di resa della trasformazione casearia. La cagliata risulta perciò più fragile e soggetta alla perdita di "polveri" (curd fines) che giustificano il maggior contenuto di proteina totale osservato nel siero quando si lavora un latte ad elevato contenuto di cellule somatiche.

\section{VARIAZIONI STAGIONALI DELLA RESA IN FORMAGGIO PARMIGIANO REGGIANO E GRANA PADANO}

Le variazioni stagionali della resa sono in stretta relazione con le variazioni stagionali di grasso e caseina, e, in misura minore, con quelle dei parametri di coagulazione. Sulle prime si riflettono in ma-

Figura 3. Latti con diverso valore di cellule somatiche (suddivisi in 5 classi) e resa a 24 ore in Parmigiano Reggiano. A lettera uguale corrispondono differenze non significative (Summer et al., 2015).

Figure 3. Milks with different somatic cell count (divided into 5 classes) and 24 hours Parmigiano Reggiano cheese yield. The same letter corresponds to not-significant differences (Summer et al., 2015).

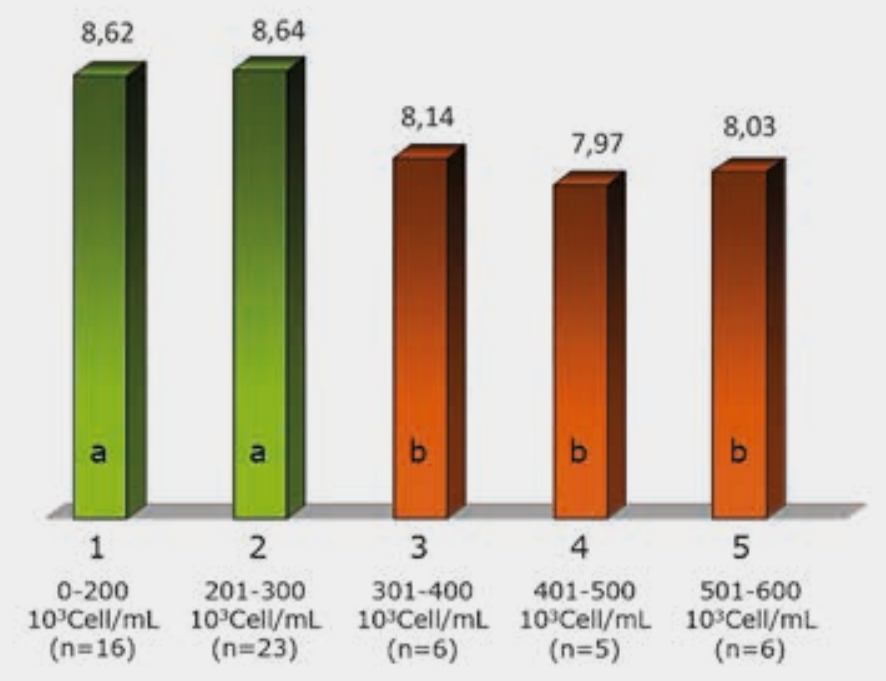


niera marcata le differenze di conduzione degli allevamenti e di gestione alimentare specifiche di ciascuna produzione casearia, sia per quanto riguarda la stagionalità dei parti, sia in riferimento al regime alimentare adottato, che in qualche produzione conserva ancora le differenze stagionali fra alimentazione verde e secca. Sul secondo fattore di variazione, ovvero quella a carico delle proprietà di coagulazione, invece, esercita un effetto diretto la temperatura ambientale, che si riflette sullo stato di benessere dell'animale e, di conseguenza sulla qualità del latte.

Le variazioni stagionali della composizione del latte, e in particolare quelle riguardanti il contenuto di proteina o di caseina, si riflettono in misura marcata sul rendimento della maggior parte delle trasformazioni casearie [14, 15, 16, 17]. Pecorari e Mariani [18], in particolare, riportano per la resa in Grana Padano valori minimi in corrispondenza dei mesi di giugno, luglio e agosto, e valori massimi nei mesi autunnali, in particolare in ottobre. Questo dato trova conferma in letteratura: anche Ozimek e Kennelly [19] evidenziano un analogo andamento nella resa in Cheddar, con valori minimi nei mesi di giugno, luglio e agosto e massimi in corrispondenza dei mesi autunnali; un risultato analogo viene riportato da Bynum e Olson [20] e Barbano e Sherbon [16], entrambi relativamente alla resa in formaggio Cheddar, e da Altiero et al. [21] per la Mozzarella di Bufala Campana; questi Autori mettono in evidenza la stretta relazione fra le variazioni stagionali di grasso e caseina e quelle della resa.

Di natura differente sono le osservazioni sperimentali di Banks e Tamime [22], che riscontrano per il contenuto di grasso valori massimi nel mese di maggio e valori minimi in dicembre e gennaio e per la caseina valori massimi in maggio e valori minimi a gennaio e novembre; di conseguenza la resa in formaggio (Cheddar), presenta valori minimi a gennaio e valori massimi a settembre e ottobre.

Da questi dati emerge chiaramente che le condizioni climatiche e lo stato fisiologico dell'animale esercitano un ruolo determinante sui contenuti di grasso e di caseina e sulle proprietà di coagulazione, fattori che a loro volta si ripercuotono sul rendimento caseario.

I primi dati sulle variazioni stagionali della resa in grana: il libro di Parisi (1966)

Parisi [23] trova le seguenti variazioni stagionali medie della resa in formaggio grana: aprile: 6,5\%; maggio: 6,8\%; giugno 7,0\%; luglio: 7,2\%; agosto: 7,5\%; settembre: 7,8\%; ottobre: $8,0 \%$; novembre: $8,2 \%$.

Anche se non vi è un'analisi statistica dei dati, è evidente che la resa va aumentando progressivamente da aprile (valore più basso $6,5 \%$ ) a novembre (valore più alto $8,2 \%$ ). È inutile sottolineare quanto le rese fossero differenti rispetto ad oggi, e quanto fosse elevata, rispetto ad oggi, la forbice di differenza fra i mesi primaverili e quelli autunnali.

Variazioni stagionali della resa in Parmigiano Reggiano: più bassa in primavera, più alta in autunno: lo studio di Pecorari e Mariani (1990)

Pecorari e Mariani [18] hanno effettuato 8 prove comparative di caseificazione a Parmigiano Reggiano, con latti a diverso contenuto di caseina.

Nella zona di produzione del Parmigiano Reggiano, la pratica della concentrazione dei parti che era in uso a quel tempo all'inizio dell'anno rendeva le variazioni stagionali della resa in formaggio particolarmente accentuate, ed in gran parte legate, appunto, all'influenza dello stato fisiologico delle vacche sul contenuto in caseina del latte. Nei mesi di gennaio, febbraio e marzo, ad esempio, i valori di contenuto in caseina del latte e, conseguentemente, quelli della resa in formaggio, risultavano tra i più bassi dell'intera annata casearia; mentre durante i mesi di settembre, ottobre e novembre, quando le vacche si trovavano nel periodo finale della lattazione, gli autori hanno registrato sia i più elevati tenori in caseina sia le più elevate rese in formaggio. La differenza, anche in questo caso, era totalmente legata alla caseina, poiché nei due periodi considerati il rapporto grasso:caseina del formaggio risultava perfettamente uguale $(0,87)$. Si può, inoltre, rilevare come durante i mesi estivi si verificava una situazione particolarmente sfavorevole, a carico sia della resa in formaggio sia della produzione di latte, molto probabilmente legata all'azione di più fattori, nell'ambito dei quali le avverse condizioni climatiche svolgevano un ruolo piuttosto importante.

\section{Variazioni stagionali della resa in Grana Padano: lo studio di Mariani et al. (1995)}

Lo studio di Mariani et al. [24] ha avuto l'obiettivo di determinare le variazioni stagionali della resa in Grana Padano. La ricerca è stata condotta presso un caseificio in provincia di Cremona. Nel corso di 3 anni consecutivi, mediante doppia lavorazione giornaliera, da 156.305 q di latte, raccolti da circa 50 allevamenti bovini di razza Frisona, sono state ottenute 31.000 forme di Grana Padano, con una resa media in formaggio pari a 7,11\% (kg di formaggio fresco per $100 \mathrm{~kg}$ di latte conferito al caseificio). I rilievi sono stati effettuati giornalmente e i dati elaborati per decade e per mese nel corso dei singoli anni e per il triennio. La resa del latte in formaggio varia in misura significativa nel corso dell'anno $(\mathrm{P}<0,0001)$. Le rese più elevate $\mathrm{si}$ registrano in ottobre $(7,33 \%)$ e in novembre $(7,30 \%)$, mesi durante i quali il latte risulta, molto probabilmente, più ricco di caseina e di 
grasso, soprattutto per l'effetto favorevole di uno stadio di lattazione mediamente più avanzato. Le rese più basse si registrano in luglio $(6,86 \%)$ e in agosto $(6,94 \%)$, mesi durante i quali il latte risulta, molto probabilmente, più povero di caseina e di grasso, soprattutto per effetto delle sfavorevoli condizioni di temperatura e di umidità relativa, entrambe elevate. Tali condizioni climatiche influiscono negativamente sullo stato metabolico-nutrizionale delle vacche, determinando anche una marcata diminuzione della produzione di latte.

Variazioni stagionali della resa in Parmigiano Reggiano in tempi più recenti: lo studio di Formaggioni et al. (2004)

Obiettivo dello studio di Formaggioni et al. [25], è stato quello di registrare le variazioni stagionali della resa in Parmigiano Reggiano. I prelievi hanno avuto luogo nell'arco di due anni e mezzo in 28 differenti caseifici, situati in provincia di Parma e variamente distribuiti tra pianura, collina e montagna. Presso ogni caseificio sono stati prelevati i latti di due distinte caldaie, lavorati in parallelo. Sono stati considerati latti con contenuto differente di caseina e di grasso, allo scopo di valutare meglio gli effetti dei due parametri sulla resa. Le lavorazioni sono state effettuate in diversi periodi stagionali.

Sono stati effettuati rilievi ponderali su latte della sera, latte magro, panna, latte intero del mattino e latte in caldaia. È stato, inoltre, rile- vato il peso delle forme a 24 ore, 6 mesi, 18 mesi e, in alcuni casi, a 24 mesi dalla produzione.

La maggior parte dei costituenti del latte e le sue principali caratteristiche hanno fatto registrare importanti variazioni stagionali, in alcuni casi statisticamente significative. Per quanto riguarda le variazioni stagionali della resa, l'analisi è stata limitata ai valori di resa a $24 \mathrm{~h}$ e della resa a 6 mesi. La resa casearia del latte in caldaia è risultata significativamente più elevata in autunno, sia a 24 ore $(8,52 \% ; \mathrm{P}<0,05)$ sia a 6 mesi di stagionatura $(7,77 \% ; P<0,01)$. Lo stesso vale per $i$ valori della resa riferita al latte conferito al caseificio ("latte massa"): $8,33 \%$ a $24 \mathrm{~h}(\mathrm{P}<0,01)$ e $7,60 \%$ a 6 mesi $(P<0,01)$. All'età di 6 mesi il formaggio prodotto in autunno, in maniera non dissimile da quello prodotto in estate, ha fatto registrare un minor calo percentuale di peso: $8,73 \%$ autunno e $8,54 \%$ estate, contro $9,39 \%$ in inverno e $9,48 \%$ in primavera $(\mathrm{P}<0,0001)$; questo fenomeno, verosimilmente, è legato al fatto che il formaggio prodotto in inverno e in primavera, durante i primi 6 mesi di vita va incontro al caldo del periodo successivo estivo.

Le proprietà di coagulazione presamica hanno manifestato variazioni stagionali piuttosto marcate; i valori dei parametri tempo di coagulazione, tempo di rassodamento e consistenza del coagulo sono risultati decisamente peggiori durante i mesi estivi.

\section{CONCLUSIONI}

In conclusione, il latte con più di $400.000 \mathrm{cell} / \mathrm{mL}$ ha fatto registrare una resa in Parmigiano Reggiano a 24 mesi di stagionatura significativamente più bassa, sia riferita al latte in caldaia (una perdita dell' $8,10 \%$ ), che riferita al latte conferito in caseificio (latte del mattino + latte della sera intero; una perdita del 7,61\%). Anche distribuendo in classi i latti con diverso valore di cellule somatiche, si è potuto riscontrare che i latti appartenenti alle classi con cellule somatiche più elevate fornivano una resa in Parmigiano Reggiano più bassa. In particolare, un calo significativo della resa a 24 ore è stato osservato nelle classi 3, 4 e 5, ovvero quando il valore di cellule somatiche del latte della sera superava le 300.000 cellule/mL, quindi ancora prima che il latte raggiungesse il valore di 400.000 cellule/mL (limite legale europeo). Tali differenze nella resa in formaggio possono comportare perdite economiche rilevanti, se si aggiungono ad esse anche la minore produzione di latte e le cure veterinarie a cui vanno soggette le bovine con un elevato contenuto di cellule somatiche.

Le perdite in termini di resa casearia possono essere spiegate a più livelli: nel latte con elevato contenuto di cellule somatiche, la caseina risulta presente in minore quantità (minore sintesi) e in peggiori condizioni, in quanto parzialmente idrolizzata dalla plasmina e dagli enzimi rilasciati dai globuli bianchi; il peggioramento delle proprietà di coagulazione dà luogo ad un reticolo con caratteristiche peggiori che trattiene con più difficoltà le componenti (soprattutto il grasso) durante la coagulazione stessa e nel corso della spinatura, dando fenomeni di slattamento; si formano granuli di dimensione eterogenea, anche molto piccoli e completamente disidratati, che perdono il loro potere adesivo e non si fondono con i resto della massa caseosa, andando a costituire le polveri di cagliata che si perdono nel siero.

Relativamente alle variazioni stagionale della resa, possiamo dire che già nel 1966 veniva registrata una resa più elevata in formaggio grana nei mesi autunnali. Questo trend è stato confermato anche nel 1990 per il Parmigiano reggiano e nel 1995 per il Grana Padano. Durante i mesi estivi si registrava spesso la resa più bassa: evidentemente si verificava in tali mesi una situazione 
particolarmente sfavorevole, a carico sia della resa in formaggio sia della produzione di latte, molto probabilmente legata all'azione di più fattori, nell'ambito dei quali le avverse condizioni climatiche di temperatura e di umidità relativa, entrambe elevate, svolgevano un ruolo piuttosto importante. Al contrario, in ottobre e in novembre il latte risultava, molto probabilmente, più ricco di caseina e di grasso, anche spesso per l'effetto favorevole di uno stadio di lattazione mediamente più avanzato.

Tale trend si è leggermente modificato nel quadro mostrato da una ricerca più recente, risalente al 2004. Infatti gli autori riscon- trano i valori più alti di resa in Parmigiano Reggiano sempre nei mesi autunnali, ma i più bassi in inverno e primavera. Evidentemente le condizioni ambientali nel periodo estivo, nelle moderne stalle con ventilatori, e con una più profonda conoscenza e attenzione per il benessere animale, esercitano il loro impatto in misura minore che in passato sulle caratteristiche metaboliche delle bovine e sulla composizione del latte. Nei formaggi prodotti in inverno e in primavera, si sono invece registrati i maggiori cali peso per evaporazione, perché le forme, nel momento della maggiore perdita di peso, andavano incontro al periodo estivo.

CONFLITTO DI INTERESSE

Non esistono conflitti di interesse di ordine economico o di altro tipo sull'articolo presentato.

\section{BIBLIOGRAFIA}

1. Moussauoi F, Michelutti I, Le Roux Y, Laurent F. Mechanisms involved in milk endogenous proteolysis induced by a lipopolysaccharide experimental mastitis. J Dairy Sci 2002;85:2562-2570.

2. Mazal G, Vianna PCB, Santos MV, Gigante ML. Effect of somatic cell count on Prato cheese composition. J Dairy Sci 2007;90:630-636.

3. Houben EHP, Huirne RBM, Dijkhuizen AA, Kristensen AR. Optimal replacement of mastitic cows determined by a hierarchic markov process. J Dairy Sci 1994;77:2975-2993.

4. Auldist MJ, Hubble IB. Effects of mastitis on raw milk and dairy products. Aust J Dairy Technol 1998;53:28-36.

5. De los Campos G, Gianola D, Heringstad B. A structural equation model for describing relationships between somatic cell score and milk yield in first-lactation dairy cows. J Dairy Sci 2006;89:4445-4455.

6. Lescourret F, Coulon JB. Modelling the impact of mastitis on milk production by dairy cows. J Dairy Sci 1994;77:2289-2301.

7. Politis I, Ng-Kwai-Hang KF. Association between somatic cell count of milk and cheese-yielding capacity. J Dairy Sci 1988;71:1720-1727.

8. O'Brien B, Meaney WJ, McDonagh D, Kelly A. Influence of somatic cell count interval on composition and processing characteristics of milk from cows in late lactation. Aust J Dairy Technol 2001;56:213-218.

9. Barbano DM, Rasmussen RR, Lynch JM. Influence of milk somatic cell count and milk age on cheese yield J Dairy Sci 1991;74:369-388.

10. Verdier-Metz I, Coulon JB, Pradel P. Relationship between milk fat and protein contents and cheese yield. Anim Res 2001;50:365-371.

11. Summer A, Franceschi P, Malacarne M, Formaggioni P, Sandri S, Pecorari M, Mariani P. Influenza del contenuto in cellule somatiche del latte sulla resa in Parmigiano-Reggiano. Buiatria, Journal of the Italian Association for Buiatrics, 2010;5(1):31-37.

12. Costanzo A, Franceschi P, Formaggioni P, Malacarne M, Di Frangia F, Summer A. Influenza del contenuto di cellule somatiche del latte su resa $e$ perdite di lavorazione nella produzione del Parmigiano Reggiano. Sci Tecn Latt-cas 2015;66 (5-6):125-135.

13. Summer A, Franceschi $P$, Formaggioni $P$, Malacarne M. Influence of milk somatic cell content on Parmigiano-Reggiano cheese yield. J Dairy Res 2015;82(2):222-227.

14. Cusmano I, Russo F, Tromellini P. Sul rapporto grasso-caseina del latte in caldaia da trasformare in formaggio Parmigiano-Reggiano. Latte 1963;37:456-464.

15. Labuschagne JH. Influenza della composizione chimica del latte sulla qualità del formaggio. Latte 1982;7:294-298.

16. Barbano DM, Sherbon JW. Cheddar cheese yields in New York. J Dairy Sci 1984;67:1873-1883.

17. Gilles J, Lawrence RC. The yield of cheese. NZJ Dairy Sci Technol 1985;20:205-214.

18. Pecorari M, Mariani P. Caseina, attitudine alla coagulazione del latte, resa e qualità del formaggio. Sci Tecn Latt-cas 1990;41:225-244.

19. Ozimek L, Kennelly J. The effect of seasonal and regional variation in milk composition on potential cheese yield. In "Cheese yield, factors affecting its control" IDF Seminar, Cork (Ireland), 1993;95-100.

20. Bynum DG, Olson NE. Influence of curd firmness at cutting on Cheddar cheese yield and recovery of milk constituents. J Dairy Sci 1982;65:2281-2290.

21. Altiero V, Moio L, Addeo F. Previsione della resa in Mozzarella sulla base del contenuto in grasso e proteine del latte di Bufala. Sci Tecn Lattcas 1989;40:425-433.

22. Banks JM, Tamime AY. Seasonal trends in the efficiency of recovery of milk fat and casein in cheese manufacture. J Soc Dairy Technol 1987;40:64- 66 .

23. Parisi O. II formaggio Grana. Ed. Stem Mucchi, Modena, 1966.

24. Mariani P, Summer A, Maffezzoli F, Zanzucchi G. Variazioni stagionali della resa del latte in formaggio Grana Padano. Ann Fac Medic Vet Univ Parma 1995;15:159-166.

25. Formaggioni P, Pecorari M, Franceschi P, Sabbioni A, Malacarne M, Summer A, Mariani P. Composizione chimica, coagulazione presamica e resa del latte nella caseificazione a Parmigiano-Reggiano: variazioni stagionali. Ann Fac Medic Vet Univ Parma 2004;24:327-343. 